\title{
Comparative Study of Fat body Lipid Alterations in White Grub adults of Leucopholis Lepidophora and Oryctes Rhinoceros
}

\author{
S. B More ${ }^{1}$ and L. P. Lanka ${ }^{2}$ \\ ${ }^{l}$ Department of Zoology, P.V.P. Mahavidyalaya, Kavathe Mahankal, Dist. Sangli (M.S.), India 416405 \\ ${ }^{2}$ Department of Zoology, Devchand College Arjunnagar, Nipini
}

\begin{abstract}
The fat body plays major roles in the life of insects. It is a dynamic tissue involved in multiple metabolic functions. One of these functions is to store and release energy in response to the energy demands of the insect. Insects store energy reserves in the form of glycogen and triglycerides in the adipocytes, The neutral lipids $(N L)$ and phospholipids $(P L)$ with their constituents were studied in the fat body of male and female adults of Leucopholis lepidophora and Oryctes rhinoceros by employing thin layer chromatography (TLC) and bioassay technique. Comparatively O. rhinoceros exhibits more lipids than L .lepidophora. The quantity of neutral lipids in male and female fat bodies of former and later species was measured 30.12, 45.212 and 49.221. $63.894 \mathrm{mg} / \mathrm{gm}$ wet weight of tissues respectively. The phospholipid values in male and female fat bodies of former species were 20.663, 14.798 and $23.266,17.349 \mathrm{mg} / \mathrm{gm}$ wet weight of tissues respectively. The NL: $P L$ ratio in male and female adult fat bodies of former species was 2: I and 3:1 whereas in later species it becomes 2:1 and 4:1. This indicated that the neutral lipids were dominated over the phospholipids in both species. . The neutral lipids found in six forms. Triacylglycerol was the main component, monoacylglycerol, diacylglycerol, cholesterol, was moderate and cholesterol ester and free fatty acids low in quantity. Phospholipids exhibited seven constituents; phosphatidyl-choline and phosphatidyl-ethanolamine high in concentration, Lysophosphatidyl-choline and sphingomyelin moderate in concentration, and phosphatidylinositol, phosphatidyl-serine and phosphatidic acid low in quantity.
\end{abstract}

Keywords: lipids, Fat body. Thin layer chromatography, Leucopholis lepidophora and Oryctes rhinoceros.

\section{Introduction}

The white grub have become known polyhagus and found in a particular type of agro ecosystem. In India Lefray , (1900) Ghosh (1937) studied the white grubs of sugarcane . White grubs are most destructive insect pest all over the world. Adult Oryctes rhinoceros is the pest of coconut trees; responsible for causing considerable reduction in yields. Lipids are bio-chemically important components of insect. Lipid performs a variety of functions in insect physiology. Triacylglycerol is utilized for biological energy (Downer, 1997). The insect fat body plays an essential role in energy storage and utilization. It is an organ of great biosynthetic and metabolic activity (Law and Wells, 1989). Fatty acids stored in the lipid droplets of the fat body are mobilized for a number of purposes, to flight muscles, in the form of diglyceride; to the ovaries; and the overall maintenance of the metabolic activity. According to Olofsson et.al 2009 and Soulages et.al, 1994 Fat body lipids are commonly secreted into the hemolymph as diacylglycerol, which is transported to the tissues by the insect lipoprotein, lipophorin The utilization of lipids in insects has been reviewed on several occasions (Beenakkers, et.al 1985, Canavoso et.al , 2001, Downer and Matthews1997). Cheng et.al 2006 and Jiang et.al 2005 reported that; Fat body is a multifunctional organ in D. melanogaster and Bombyx mori . Fat body cells control the synthesis and utilization of energy reserves. ( Keeley, 1985). The fat body is structurally heterogeneous and functions of the fat body are present all over the tissue (Haunerland and Shirk, 1995 and Jensen and Borgesen, 2000. Dean et.al, 1985 and Athenstaedt and Daum, 2006 stated that triglycerides are the major component of the lipid droplets, and Fatty acids stored as triglyceride can be used for energy production. Triglyceride is stored in an anhydrous form, and also has a higher caloric content. The conversion of carbohydrates, a major component of the insect diet, to lipid in the fat body is well documented ( Briegel, 1990; Hines and Smith, 1963; Inajaki Yamshita, 1986; Kim and Rulifson1985, and Van Der Horst 2002). Dean 1985 reported that fat body adipocytes cells are able to store large amounts of triglycerides. A lipid droplet consists of a core of neutral lipids (triglyceride and cholesterol esters) surrounded by a monolayer of phospholipid and cholesterol ( Bickel et.al 2009 and Brassaemle, 2007). The significance of phospholipids with PC and PE was explained by Locke and Krishnan 1071.

\section{Materials and Methods}

The prepupae and adults of $O$. rhinoceros were collected from dunghill of Agriculture College, near the Shivaji University, campus Kolhapur, (M.S.). They were reared at laboratory and adults were used for fat body lipid extraction; wherwas, male and female adults of L. lepidophora were collected from sugarcane field 
of Sangrul village (Dist- Kolhapur, M.S.) They kept in laboratory for acclimatization. The adults were dissected and fat bodies were accurately weighed and used for lipid extraction.

\section{1) Extraction of Lipids}

The fat body of male and female adults of both species were weighed and homogenized with $20 \mathrm{ml}$ of chloroform-methanol $(2: 1 \mathrm{v} / \mathrm{v})$ at room temperature. Five male and five female adults were taken for the present investigation the homogenates were allowed to stand for 2-3 hours at $4^{\circ} \mathrm{c}$ and filtered. The filtrate was washed according to Floch et.al; (1957) and evaporated in vacuum at $40^{\circ} \mathrm{c}$. The lipid samples were weighed and preserved at $-20^{\circ} \mathrm{c}$ until further use. The total lipid in the sample was determined gravimetrically.

\section{2) Separation of Neutral Lipids and Phospholipids}

The neutral lipids and phospholipids were separated by thin layer chromatography (TLC) using silica gel $\mathrm{G}$ and about 200 mesh containing CaSo4, as a binder, (E Merck Germany). The TLC plates $(20$ X $20 \mathrm{~cm})$ were prepared according to Wagner et.al (1961). The known quantities of samples dissolved in chloroform were applied with Hamilton's micro syringe (No.8206-B) on activated plates. For neutral lipid the plates were developed in hexane (B.P. $65-70^{\circ} \mathrm{c}$ ) diethyl ether - acetic acid $(85: 15: 2 \mathrm{v} / \mathrm{v})$ as recommended by Gloster and Flecter (1966). The phospholipid plates were developed in chloroform-methanol-ammonia $(115: 45: 5 \mathrm{v} / \mathrm{v})$ as recommended by Barwal and Kalra (1988). The standards of neutral lipids and phospholipids (Sigma,U.S.A.) were co-chromatographed in each respective run and then plates were kept in iodine chamber for identification of individual spots of lipids.

\section{3) Estimation of Neutral Lipids and Phospholipids.}

The iodine was allowed to evaporate and the silica gel from the individual spots of glycerides was scraped and eluted in $1 \mathrm{ml}$ of diethyl-ether and assayed according to Viogue and Holman (1962). The cholesterol and its ester were estimated according to Abell et.al (1952). The rest of the neutral lipid components were assayed titrometrically by the method of Skipski et.al (1967). The phospholipid was determined by the method of Marinetti (1967).

\section{i) Neutral Lipids}

\section{Results}

The TLC separation of various neutral lipid components are illustrated in plate 1 Fig. A; whereas, Table No.1 exhibits quantitative variations in the neutral lipid components. The neutral lipids in male and female fat bodies of L. lepidophora were measured 30.12, 45.212 and in O.rhinoceros were $49.221 \pm 2.15$ and $63.894 \pm 3.23 \mathrm{mg} / \mathrm{gm}$. wet weight of tissues respectively. The neutral lipids consists of six components; of these triacylglycerol (TG) being the major component. Monoacylglycerol (MG), diacylglycerol (DG) and cholesterol (CHO) were found moderate in concentration; whereas free fatty acids (FFA) and cholesterol ester (CE) were occurred low in quantities. The TG concentration in fat bodies of male and female of former species was $21.43,32.23$ and in later species 40.42 and $50.05 \mathrm{mg} / \mathrm{gm}$ wet weight of tissues respectively.

\section{ii) Phospholipids}

The phospholipids are illustrated in plate No.1, Fig B and Table 2. The phospholipids in male and female fat bodies in former species were measured 20.663, 14,798 and in later species were $23.266 \pm 0.76$ and $17.349 \pm 0.93 \mathrm{mg} / \mathrm{gm}$. wet weight of tissues respectively. The TLC separation of phospholipids included following constituents; phosphatidyl-choline (PC), phosphatidyl-ethanolamine (PE), Lysophosphatidyl-choline (LPC), sphingomyelin (SPG), phosphatidyl-inositol (PI), phosphatidyl-serine (PS) and phosphatidic acid (PA). Among the phospholipids PC and PE were predominant in both species. In male and female fat bodies of former species they were measure about $340.2 \pm 3.37,325.2 \pm 10.01$ and in later species $382.1 \pm 4.57,360.3 \pm$ $13.01 \mu \mathrm{g}-\mathrm{P} / \mathrm{gm}$ and $285.1 \pm 13.9,280.5 \pm 13.7 \mu \mathrm{g}-\mathrm{P} / \mathrm{gm}$ wet weight of tissues respectively. The LPC and SPG were estimated in moderate concentration, whereas PI, PS and PA less in amount. 


\section{PLA TE 1}
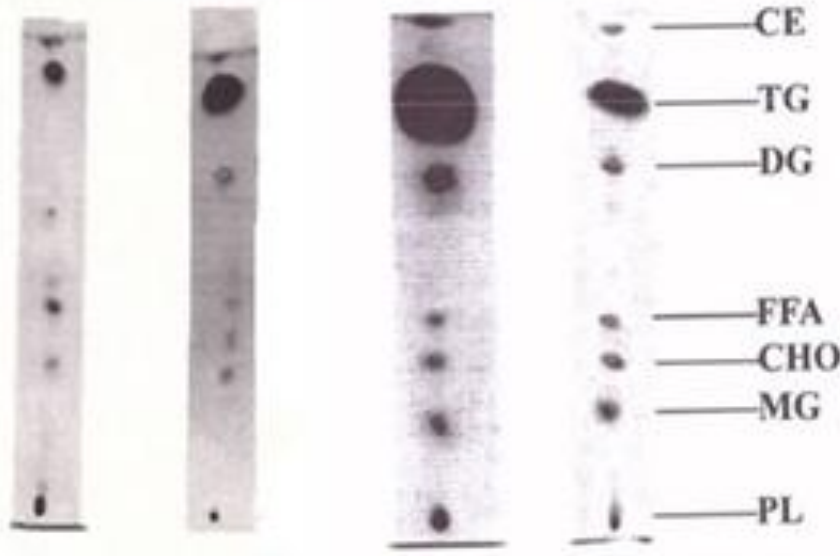

FIG-A
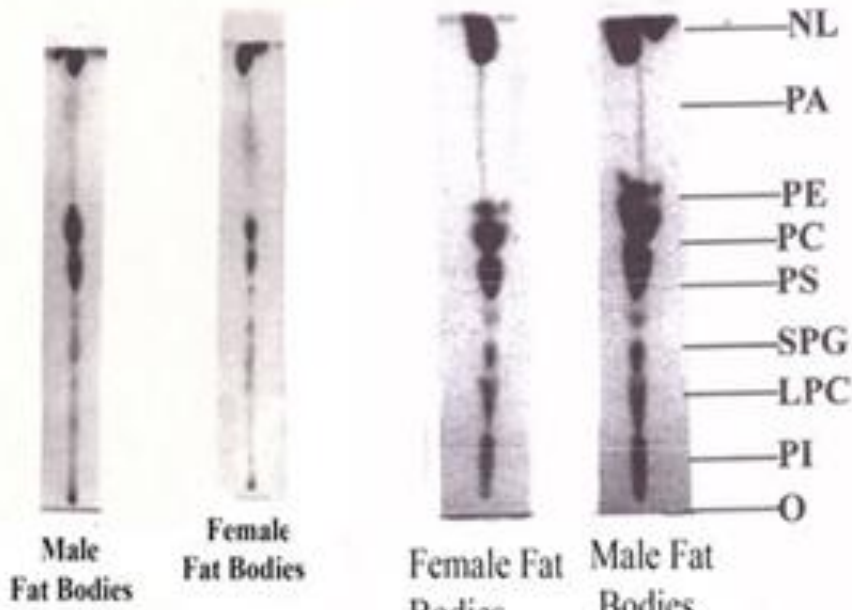

\section{Female Fat Male Fat \\ Bodies Bodies}

FIG.-B

Lexcopholis lepidaphora

Oyctesthinoceros

Table No. 1: Alterations in total lipids, neutral lipids and its components in the male and female Fat Bodies of Leucopholis lepidophora and Oryctes rhinoceros

\begin{tabular}{|l|l|l|l|l|}
\hline \multirow{2}{*}{ Lipid Type } & \multicolumn{2}{|c|}{ Fat Bodies of Leucopholis lepidophora } & \multicolumn{2}{c|}{ Fat Bodies of Oryctes rhinoceros } \\
\cline { 2 - 5 } & Male Fat Bodies & Female Fat Bodies & Male Fat Bodies & Female Fat Bodies \\
\hline Total Lipids & $60.283 \pm 2.64$ & $60.014 \pm 2.60$ & $72.487 \pm 2.64$ & $81.894 \pm 3.60$ \\
\hline Neutral Lipids & $30.12 \pm 1.01$ & $45.212 \pm 2.23$ & $49.221 \pm 2.15$ & $63.894 \pm 3.23$ \\
\hline MG & $1.526 \pm 0.16$ & $4.325 \pm 0.18$ & $1.615 \pm 0.15$ & $3.335 \pm 0.16$ \\
\hline CHO & $2.833 \pm 0.10$ & $1.783 \pm 0.15$ & $1.826 \pm 0.09$ & $01.868 \pm 0.16$ \\
\hline FFA & $0.781 \pm 0.04$ & $2.318 \pm 0.07$ & $0.591 \pm 0.04$ & $2.639 \pm 0.08$ \\
\hline DG & $2.848 \pm 0.17$ & $3.424 \pm 0.03$ & $3.867 \pm 0.16$ & $4.834 \pm 0.06$ \\
\hline TG & $21.43 \pm 1.80$ & $32.23 \pm 1.20$ & $40.42 \pm 2.832$ & $50.05 \pm 1.60$ \\
\hline CE & $0.702 \pm 0.04$ & $1.132 \pm 0.11$ & $0.902 \pm 0.05$ & $1.168 \pm 0.12$ \\
\hline
\end{tabular}

The values for total lipids, neutral lipids and its components are expressed as $\mathrm{mg} / \mathrm{gm}$. wet weight of tissues.

Table No. 2: Alterations in phospholipids and its constituents in male and female Fat Bodies of Leucopholis lepidophora and Oryctes rhinoceros.

\begin{tabular}{|c|c|c|c|c|}
\hline \multirow{2}{*}{ Lipid Type } & \multicolumn{2}{|c|}{ Fat Bodies of Leucopholis lepidophora } & \multicolumn{2}{c|}{ Fat Bodies of Oryctes rhinoceros } \\
\cline { 2 - 5 } & Male Fat Bodies & Female Fat Bodies & Male Fat Bodies & Female Fat Bodies \\
\hline Phospholipids & $20.663 \pm 0.66$ & $14.798 \pm 0.73$ & $23.266 \pm 0.76$ & $17.349 \pm 0.93$ \\
\hline PI & $20.60 \pm 0.65$ & $10.70 \pm 0.32$ & $25.70 \pm 0.75$ & $20.70 \pm 0.62$ \\
\hline LPC & $56.21 \pm 2.31$ & $25.74 \pm 2.01$ & $66.21 \pm 2.76$ & $36.47 \pm 2.12$ \\
\hline G & $49.23 \pm 2.16$ & $30.22 \pm 1.23$ & $57.31 \pm 2.56$ & $33.22 \pm 1.73$ \\
\hline
\end{tabular}


Comparative Study of Fat body Lipid Alterations in White Grub adults of Leucopholis Lepidophora...

\begin{tabular}{|l|l|l|l|l|}
\hline & & & & \\
\hline & $14.18 \pm 0.65$ & $13.24 \pm 0.42$ & $16.18 \pm 0.75$ & $17.24 \pm 0.51$ \\
\hline & $340.2 \pm 3.37$ & $250.1 \pm 10.9$ & $382.1 \pm 4.57$ & $285.1 \pm 13.9$ \\
\hline & $325.2 \pm 10.01$ & $246.2 \pm 10.5$ & $360.3 \pm 13.01$ & $280.5 \pm 13.7$ \\
\hline & $20.87 \pm 0.53$ & $15.70 \pm 1.01$ & $22.87 \pm 0.64$ & $20.70 \pm 1.03$ \\
\hline
\end{tabular}

The values of phospholipids are expressed as $\mathrm{mg} / \mathrm{gm}$. wet weight of tissues; whereas, values of individual constituents are expressed as $\mu \mathrm{g}-\mathrm{P} / \mathrm{gm}$. wet weight of tissues.

\section{Discussion}

It is significantly found that, the male fat bodies of L. lepidophora and O. rhinoceros exhibited low concentration of lipids than the female. The NL: PL ratio in male and female adult fat bodies of former species was 2: I and 3:1 whereas in later species it becomes 2:1 and 4:1.This indicated that, the neutral lipids were dominated over the phospholipids. The neutral lipids found in six forms. Triacylglycerol was the main component, monoacylglycerol, diacylglycerol, cholesterol, were moderate and cholesterol ester and free fatty acids low in quantity. Among the neutral lipids TG constitute the major component. The TG of the neutral lipids in male and female fat bodies of former and later species was $21.43,32.23$ and $40.42,50.05$ respectively. Later species constitute more triacylglycerol than former species. Triacylglycerol is high in 0 . rhinoceros

Phospholipids are expressed as $\mathrm{mg} / \mathrm{gm}$ wet weight of tissues and their values in male and female fat bodies of former and later species were 20.663, 14.798 and $23.266,17.349 \mathrm{mg} / \mathrm{gm}$, respectively. In the present investigation male fat bodies exhibited high phosphplipid contents than female fat bodies in both species. Among the phospholipids the PC and PE were major constituents. The PC: PE ratio in both species was 1:1 which indicated that the $\mathrm{PC}$ and $\mathrm{PE}$ are equal in their values. Lipid stores remain stable during the remainder of larval life and are carried over into pupae and pharate adults Inagaki and Yamashita, 1986). This is good agreement with our findings. However, much of the glycogen serves as an energy source during the postfeeding larval period, and the rest is preserved to be utilized in pupae and adults. A similar pattern in the utilization of energy reserves is reported for crickets during the final nymphal instar and the molt to the adult by Anand and Lorenz in 2008. Therefore both triglyceride and glycogen are stored in the fat body. Fat reserves are used by insects to meet their energy demand during diapause ( Hahn and Denlinger, 2007) and to fuel prolonged periods of flight. ( Beenakkers et.al; 1985). The fat body plays a major role in intermediary metabolism and it is the central storage depot of nutrients and energy reserves. Fat body energy reserves are mobilized in response to the energy demands of other tissues. Lipids always represent the major component of the fat body and the main source of metabolic fuel. According to Estela and Jose (2010) triglycerides, the major lipid form, are stored in the core of the lipid droplets; Lipid droplets are dynamic organelles whose metabolic activity is dependent on the protein components.

\section{Acknowledgements}

Author thanks to Prof. V A. Sawant Dept of Zoology Shivaji University Kolhapur for his encouragement.

\section{References}

[1] Lefroy H. M. : Ent. Memories, 11, 143-146; 1900.

[2] Ghosh G. L. : Ind. J. Agri. Sci., 7 907-931; 1937.

[3] Downer RGH, Physiological and environmental consideration in insect bioenergetics, In energy metabolism in Insects, Edited By R.G.H. Downer, 1981, 1-17, Plenum Press New York,1997.

[4] Law JH, Wells MA. Insects as biochemical models. J Biol Chem. 264:16335-38; 1989.

[5] Olofsson S-O, Boström P, Andersson L, Rurberg M, Perman J, Borén J. Lipid droplets as dynamic organelles connecting storage and efflux of lipids. Biochim Biophys Acta. 1791:448-8. 2009.

[6] Soulages JL, Wells MA. Lipophorin: the structure of an insect lipoprotein and its role in lipid transport in insects. Adv Protein Chem. 45:371-415; 1994

[7] Beenakkers AMT, Vanderhorst DJ, Vanmarrewijk WJA. Insect flight metabolism. Insect Biochem. 14:243-60;1984.

[8] Canavoso LE, Jouni ZE, Karnas KJ, Pennington JE, Wells MA. Fat metabolism in insects. Annu Rev Nutr. 21:23-46; 2001

[9] Cheng DJ, Xia QY, Zhao P, Wang ZL, Xu HF, et al. EST-based profiling and comparison of gene expression in the silkworm fat body during metamorphosis. Arch Insect Biochem Physiol. 61:10-23; 2006.

[10] Jiang Z, Wu XL, Michal JJ, McNamara JP. Pattern profiling and mapping of the fat body transcriptome in Drosophila melanogaster. Obes Res. 13:1898-904; 2005

[11] Keeley LL. Biochemistry and physiology of the insect fat body. In: Kerkut GA, Gilbert LI, editors. Comprehensive Insect Physiology, Biochemistry and Pharmacology. Vol. 3. New York: Pergamon; pp. 211-28; 1985

[12] Haunerland NH, Shirk PD. Regional and functional differentiation in the insect fat body. Annu Rev Entomol. 40:121-45; 1995

[13] Jensen PV, Borgesen LW. Regional and functional differentiation in the fat body of pharaoh's ant queens, Monomorium pharaonis (L.) Arthropod Struct Dev. 29:171-84. 2000

[14] Dean RL, Collins JV, Locke M. Structure of the fat body. In: Kerkut GA, Gilbert LI, editors. Comprehensive Insect Physiology, Biochemistry, and Pharmacology. New York: Pergamon; pp. 155-210; 1985.

[15] Athenstaedt K, Daum G. The life cycle of neutral lipids: synthesis, storage and degradation. Cell Mol Life Sci. 63:1355-69; 2006. 
[16] Briegel H. Metabolic relationship between female body size, reserves, and fecundity of Aedes aegypti. J Insect Physiol. 36:165-72; 1990.

[17] Hines WJW, Smith MJH. Some aspects of the intermediary metabolism in the desert locust, Schistocerca gregaria Forskål. J Insect Physiol. 9:463-68; 1963.

[18] Inagaki S, Yamashita O. Metabolic shift from lipogenesis to glycogenesis in the last instar larval fat body of the silkworm, Bombyx mori. Insect Biochem. 16:327-31; 1986.

[19] Kim SK, Rulifson EJ. Conserved mechanisms of glucose sensing and regulation by Drosophila corpora cardiaca cells. Nature. 431:316-20; 2004.

[20] Van der Horst DJ, van Hoof D, van Marrewijk WJ, Rodenburg KW. Alternative lipid mobilization: the insect shuttle system. Mol Cell Biochem. 239:113-19; 2002.

[21] Bickel PE, Tansey JT, Welte MA. PAT proteins, an ancient family of lipid droplet proteins that regulate cellular lipid stores. Biochim Biophys Acta.1791:419-40;2009.

[22] Brasaemle DL. Thematic review series: adipocyte biology. The perilipin family of structural lipid droplet proteins: stabilization of lipid droplets and control of lipolysis. J Lipid Res. 48:2547-59; 2007.

[23] Locke M and Krishnan N, J. Phospholipids and fatty acid composition of T. granarium and biosynthesis of fatty acids during development, Comp. Biochem. Physiol., 39, 183-194; 1971.

[24] Folch, J., Lees, M. and Sloane-Stanley A simple method for the isolation and purification of the total lipid from animal tissue. G.H. J.Biol. Chem 226; 497-509; 1957.

[25] Wagner, H., Horhammer, L. and Wolfe, P.: Thin layer chromatography of phospholipids and glycolipids., Biochem., 2, 175-184; 1961.

[26] Gloster, J. Flectar, R.F., Quantitative analysis of serum lipids with thin layer chromatography., clin. Chim. Acta., 13, 235-240; 1966.

[27] Barwal, R.N. and Kalra, R.L. : Lipids lindane susceptible and resistant strains of T. castaneu., Indian J. Expt. Biol., 26, 228$234 ; 1988$.

[28] Viogue, E, and Holaman, R.T., : Quantitative estimation of esters by thin layer chromatography., J.Am.oil. chemistis. Soc., 39, 68661962.

[29] Abell, L.L., Levy, B.B., Brodie B.B., and Kendall, F.F. : A simplified method for the estimation of total cholesterol in serum and demonstration of its specificity., J. Biol. Chem. 195, 357-359, 1952.

[30] Skipski, V.P., Barclay, M., Reichman, E.S. and Good, J.J., : Biolchem, Biophys. Acta., 137, 801967.

[31] Marintti, G.V., : ed. "Lipid Chromatography Analysis”. Vol I Marcel Dekker. Inc., New York; 1967.

[32] [Anand AN, Lorenz MW. Age-dependent changes of fat body stores and the regulation of fat body lipid synthesis and mobilisation by adipokinetic hormone in the last larval instar of the cricket, Gryllus bimaculatus. J Insect Physiol. 54:1404-12, 2008

[33] Hahn DA, Denlinger DL. Meeting the energetic demands of insect diapause: nutrient storage and utilization. J Insect Physiol. $53: 760-73 ; 2007$.

[34] Beenakkers AM, Van der Horst DJ, Van Marrewijk WJ. Insect lipids and lipoproteins, and their role in physiological processes. Prog Lipid Res. 24:19-67. 1985.

[35] Estela L. Arrese and Jose L. Soulages : Insect fat body : Energy, Metabolism and Regulation, Annu Rev Entomol. 55: 207-225; 2010 . 